\title{
The Effect of Stress, Diet AND ANalytical Methods on the LEVELS OF CoRTICOID Metabolites
}

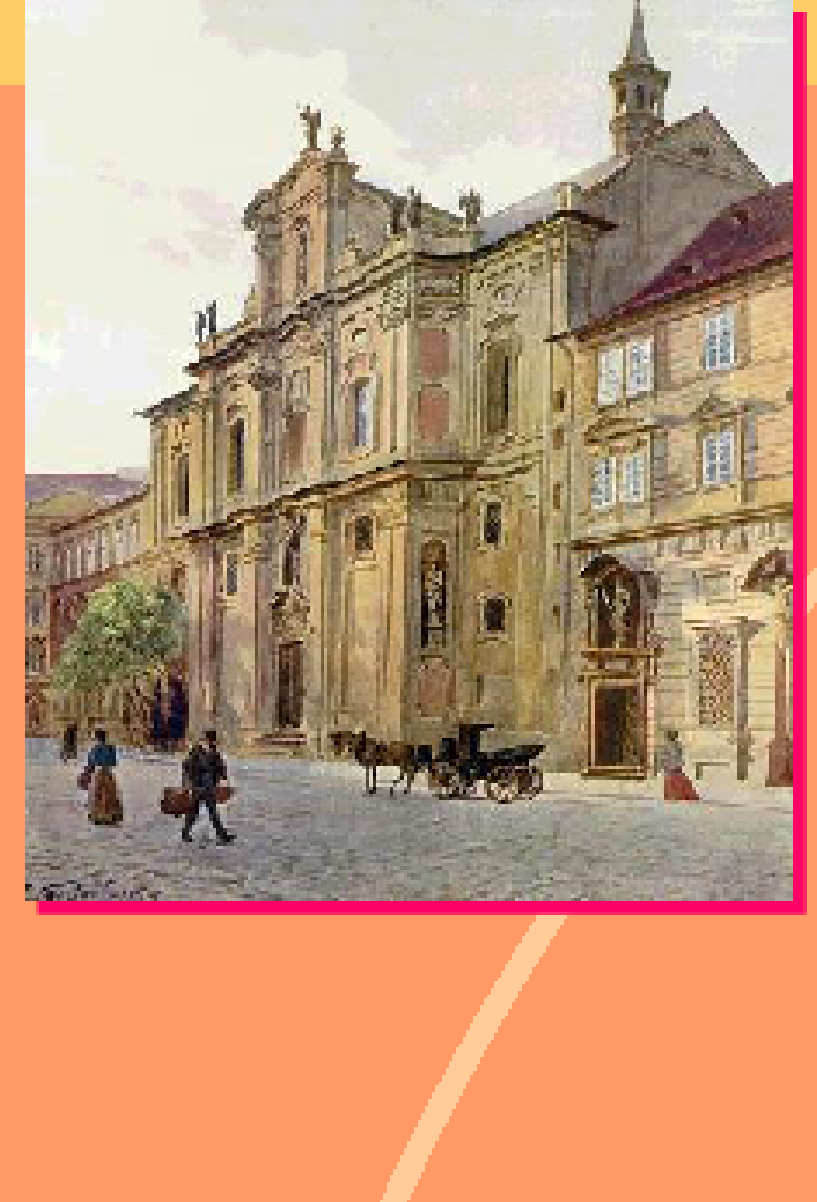

\section{Michaela Dušková, Lucie Sosvorová, Jana Vitků, Hana Jandíková, Tereza Chlupáčová, Jose De Cordeiro, Luboslav Stárka}

Institute of Endocrinology, Prague

\section{Introduction}

Measuring plasma cortisol levels is a common task of clinical biochemistry laboratories, and not just when ordered by endocrinologists. However, in the pre-analytical phase there are various influences that can distort the information sought These influences include stress brought about by the sampling, poorly-timed sampling, not taking the sampling after fasting, and choosing a method that is incompatible with the desired aims. Errors in the pre-analytical phase significantly complicate the interpretation of results, and could lead to an incorrect diagnosis.

\section{Objectives}

The aim of this study

Elucidate optimal conditions for blood sampling as well as the choice of analytical methods for measuring of corticoids.

\section{Methods}

The study was performed using 10 healthy women of reproductive age in the follicular phase of their cycle (days $1-7$ after menstruation). The average age was $33.6 \pm 2.56$ years and average BMI $25.06 \pm 1.3$. The women had no chronic diseases, were non-smokers, and did not use hormonal contraceptives or any other medications. Before starting the study, they were advised to maintain a balanced

Each woman passed two tests during two consecutive menstrual cycles:

1) Stimulation test by stress and food

g). The test started at 10:00, when cannula was inserted into the forearm or cubital vein. The time schedule - according to which the blood was drawn - was: $10: 00,10: 15,10: 30,10: 45$ and 11:00.
After the blood sampling at 12 o c'clock, the participants of this part of the study received lunch (beef broth soup, turkey, potato dumplings, and sauerkraut; total content of the lunch was $679 \mathrm{kcal}$, total protein content: $45.55 \mathrm{~g}$, total carbohydrates: 100.4 $\mathrm{g}$, total fat: 11.5 $\mathrm{g}$ ). The lunch was

Blank test

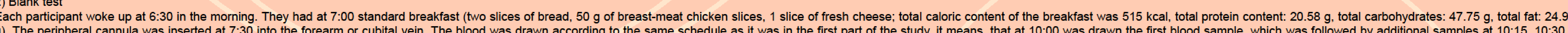
10:45 and 11:00. In contrast to the stimulation test, in the blank test participants did not have any lunch at 12 'o'clock, but they were submitted to samplings similarly as the group in the previous part of the study. The schedule of the blood drawings was: 12:00, 12:30, 13:00 and 13:30.

Blood was taken into a Vacuette Serum Clot Activator tubes (a plastic tube with a clotting activator and separation gel). Serum was obtained by centrifugation for 5 minutes at $2000 \mathrm{~g}$ at $4^{\circ} \mathrm{C}$, and stored at $-20^{\circ} \mathrm{C}$.

Stalstical anal/vses: The relationships between dependent variables and the effects of sampling time were evaluated using a repeated measures ANOVA model consisting of the following factors: Time (10:00, 10:15, 10:30, 10:45 and 11:00 for experiment 1); (11:00, 12:00, 12:30, 13:00 and (2) and Subject (explaining inter-individual variability). The ANOVA model was followed by least significant difference (LSD) multiple comparisons. To eliminate skewed data distribution and heteroscedasticity, the original data were transformed by Box-Cox transformation software Statgraphics Centurion, version XV from Statpoint Inc. (Herndon, Virging. SSA) was us

\section{Resulits}

Fig. 1

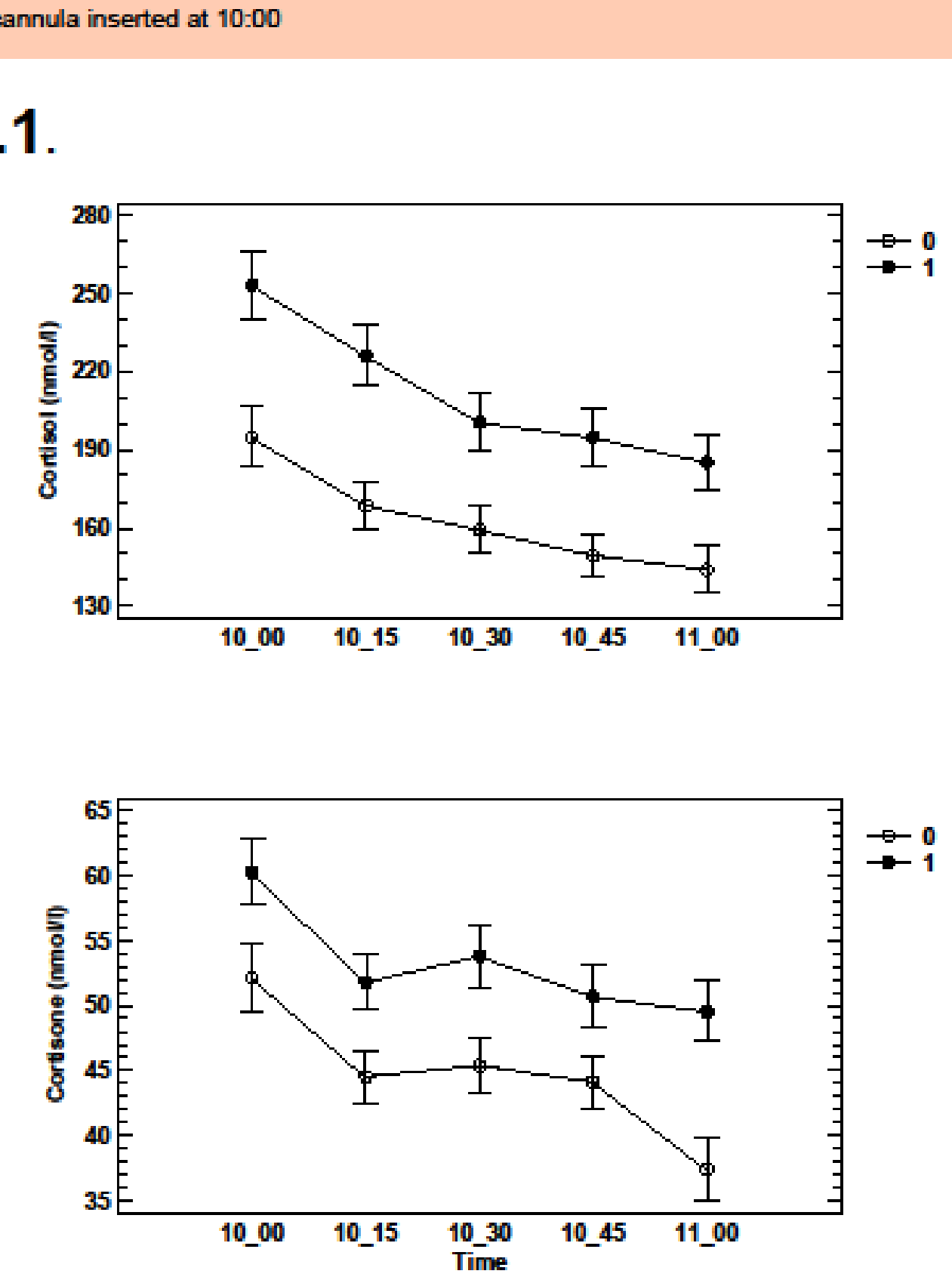

Fig.2

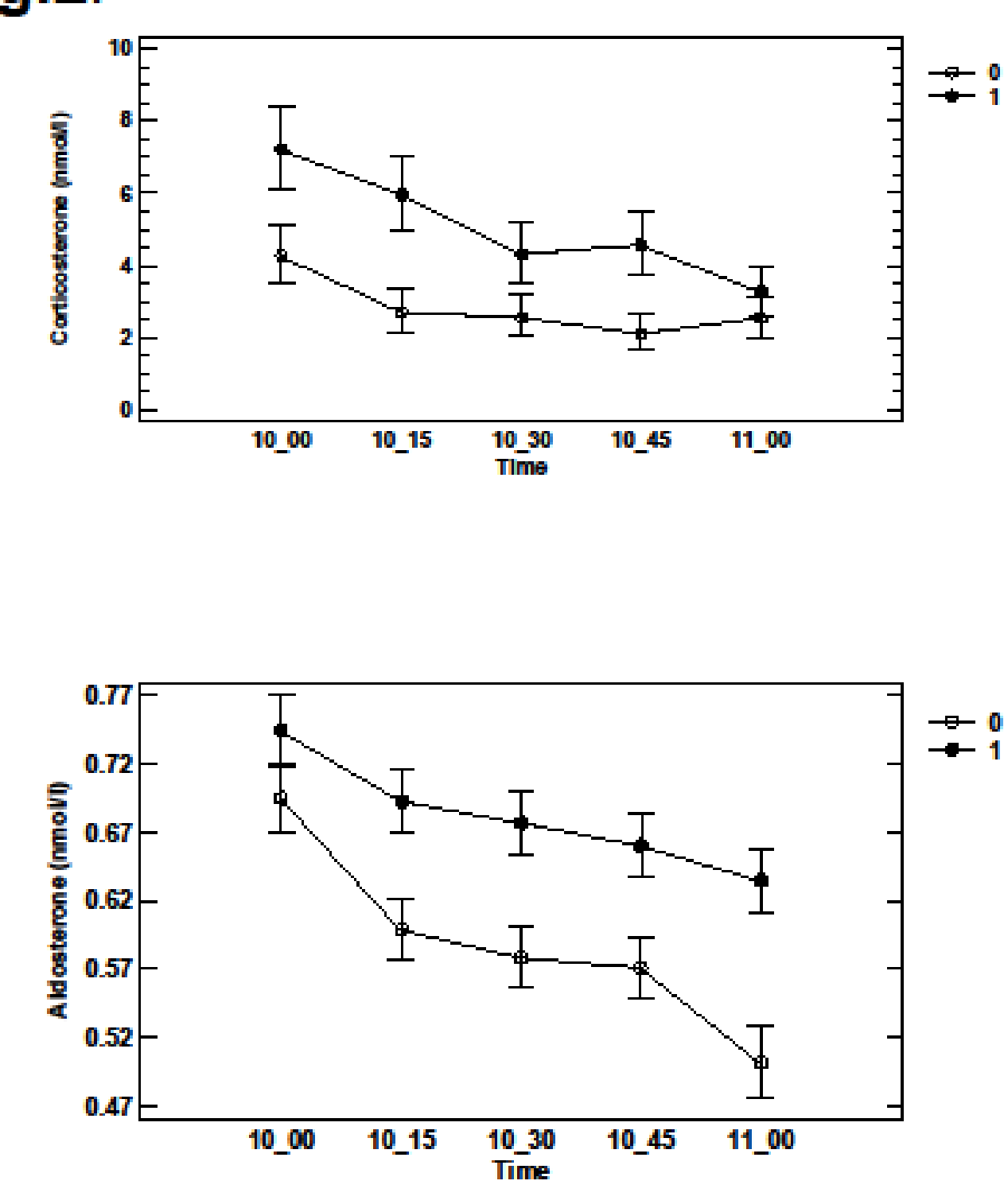

Fig.3

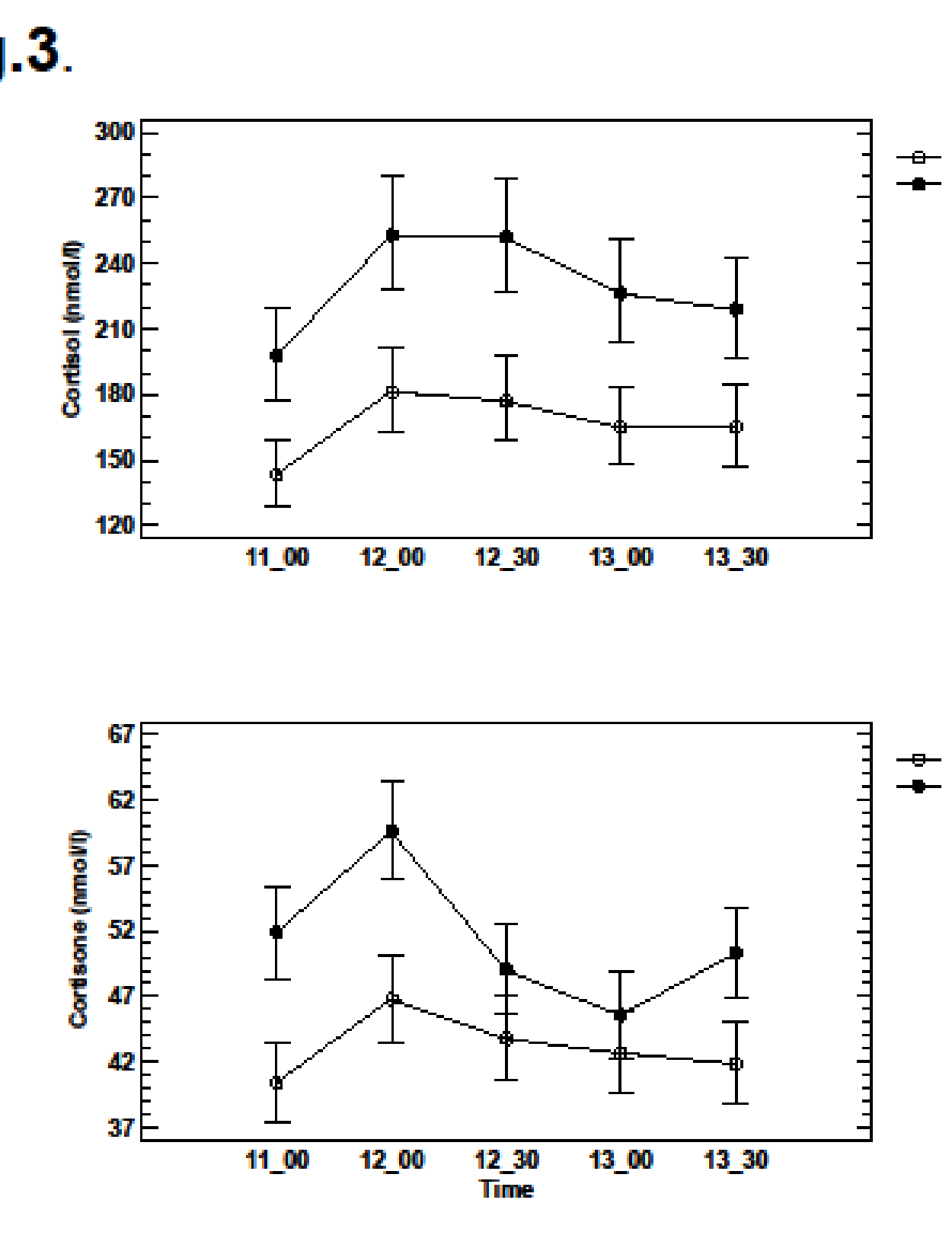

Fig.4

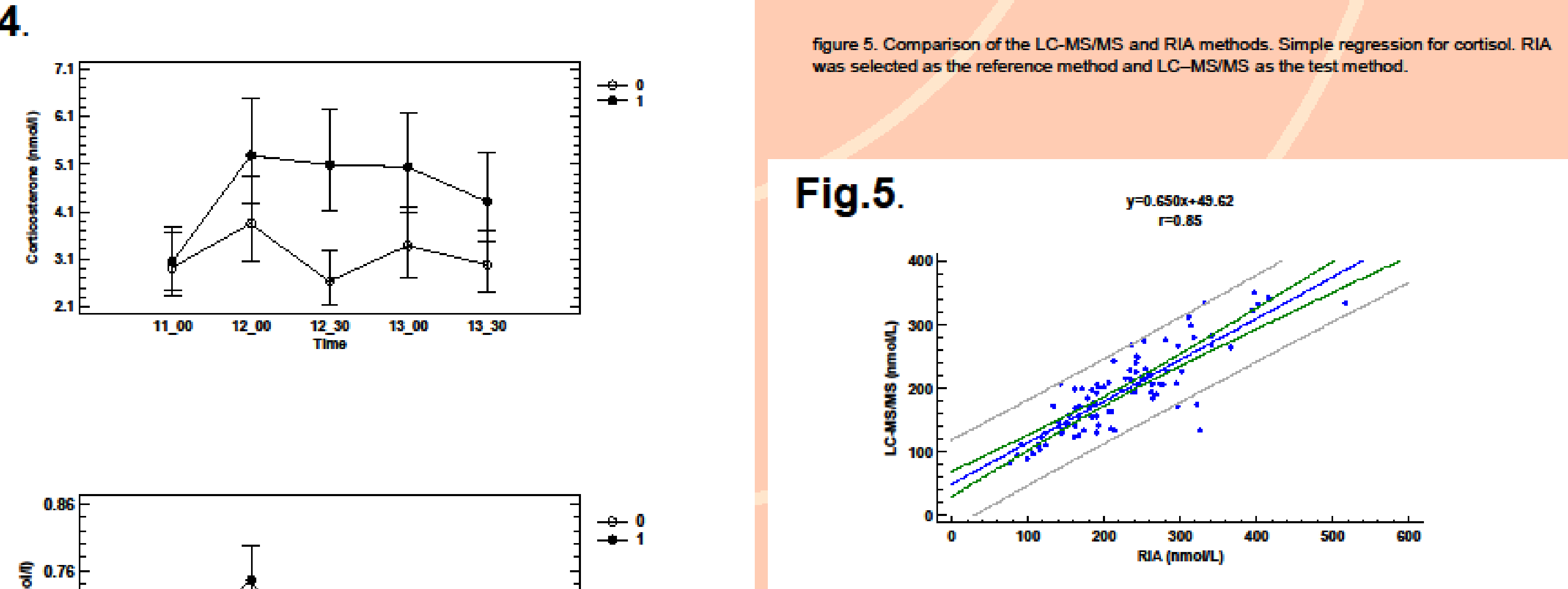

Stress:

Figure 1 shows the profile of cortisol levels when cannulation was being performed as well as when cannulation had been performed 150 minutes before the first blood sampling. Values just after inserting the cannula were significantly higher than values when calm. This reflects the fact that blood drawing can invoke minor or even fairly high stress in some patients. Higher levels of plasma cortisol lasted at least 1 hour after the first sampling. These results indicate that merely the knowledge that blood will be drawn can stimulate higher cortisol levels, not just the needle insertion itself. Cortisone levels were also increased, similarly as for cortisol (Figure 1)

Corticosterone was also increased already at the time of sampling, while increase in its metabolite aldosterone was later (Figure 2). As opposed to the other corticoids tested, the effect of stress on corticosterone disappeared after an hour (Figures 1 and 2).

The main lunchtime meal

The effects of the main meal of the day - lunch at noontime - were interesting. All corticoids tested had a marked increase between 11 and 12 o'clock, which could reflect a physiological preparation for eating as part of the circadian rhythm. After eating there was an evident decline in cortisone levels, while its precursor cortisol had rather a plateau in its decline (Figure 3). Similarly, there was an evident decline in aldosterone but a plateau in the decline of its precursor corticosterone (Figure 4).

Choice of analytical method

We compared cortisol in 90 plasma samples measured by the commercial RIA kit from Immunotech and a published LC-MS/MS method (Sosvorova et al., 2015). The RIA method, which was used as the reference method, showed strong correlation with the LC method ( $r=0.85$ ), with the regression approximated by the equation $y=0.650 x+49.62$ (Figure 5 ). The slope of the regression line indicates some overestimation of cortisol levels when using RIA.

\section{Conclusions}

Not just endocrinologists but also other specialists often require cortisol measurements from biochemical laboratories. Determining the correct levels of cortisol, especially in the differential diagnosis of hypocortisolism, hypercortisolism and normal functioning of the hypothalamic-pituitary-adrenal axis, require maintaining the proper conditions even in the pre-analytical phase of sampling. It is necessary to take into account the daily rhythm of cortisol and avoid sampling in the first hours after waking (if not specifically measuring CAR), as well as taking into account food intake and the stress of blood drawing. Finally, the choice of proper analytical method should be made with knowledge of their limitations. 